\title{
Jet Plasma-Chemical Reactor for the Conversion of Methane: the Use of Clustering
}

\author{
A. E. Zarvin, N. G. Korobeishchikov, M. D. Khodakov, V. V. Kalyada \\ Department of Applied Physics, Novosibirsk State University, Novosibirsk, Russia \\ Email: zarvin@phys.nsu.ru
}

Received 2012

\begin{abstract}
The research results of processes proceeding in supersonic jets of light hydrocarbons, activated by an electron beam are presented. It is shown, that condensation suppressed at activation by electrons in the initial stage of condensation. The developed condensation conditions mode leads to increasing of a part of heavy corpuscles in activated stream and not only owing to stimulation of condensation but because of formation of heavy hydrocarbonic molecules.
\end{abstract}

Keywords: Conversion of Methane; GTL-Production Engineering; Clusters; a Supersonic Jet; Plasmachemical Reactions; Gas Phase Reactions; an Electron Beam; a Gas Discharge

\section{Introduction}

Associated petroleum gas (a mixture of methane - more than $70 \%$, ethane, propane and butane) is traditionally burned during the oil production. Its processing is not provided in the existing technology of oil refining. As a result, approximately $10 \%$ of the hydrocarbons contained in the field, does not used in the economy only, but also contributes to further pollution of the earth's atmosphere.

Processing technology of volatile hydrocarbons exists for a long time. It's primarily based on the use of catalysts in Fischer-Tropsch process, focused on the steady-state production, and remote oil fields is almost irrelevant. It should also be borne in mind that any large-tonnage commercial product should be exported, and the only available high-performance transport of liquid products from the field - an oil pipeline.

In this situation it is extremely attractive the development of the direct technology conversion method, bypassing the stage of synthesis gas, of natural and petroleum gas to heavy hydrocarbons, which is implemented on a compact high-performance device. The resulting liquid should be suitable for pipeline transport, ie its vapor pressure must not exceed 0.5 excess air. Such a formulation of the problem points directly to the usefulness of its solution methods and approaches based on plasma-chemical processes in the gas phase: the large interaction cross section of ionized and activated particles provide an extremely high rate of any collisional processes.

\section{Plasma Chemical Technologies}

First of all, it was started investigation of the possibilities for application of discharge plasma to initiate gas-phase reactions [1-4]. In the discharge can be easily obtained active particles that provide high-speed reactions. As a result, engineers have created several versions of devices to produce hydrogen from hydrocarbons using microwave discharge plasma torch, a combination of discharge with distributed finely dispersed catalyst
[5], etc. In the 2000 method of conversion of hydrocarbons in a barrier discharge, where gas diffuses through the porous dielectric layer containing catalyst particles, was proposed [6]. A limitation of this method is the low rate of diffusion of gas as well as the need for high pressure. There are a large number of patents on the use of a glow, corona, streamer and other types of discharge, electric arc, plasma torch and so on [7-9]. However, no one has to initiate a one-step synthesis of heavy hydrocarbons on an industrial scale yet/

Using the discharge faces a number of difficulties associated with the influence of the electrodes, the background gas and the flow in a quiescent gas, or the reverse reactions in a subsonic flow, limiting the equilibrium concentration of the reaction products. These negative factors can be excluded if use the supersonic reagent jet to provide gas into reaction zone. Shock waves which are formed at the boundaries of the jet, are isolated the jet core from the influence of background gas. Freezing of the reverse reactions is provided by a sharp drop in density in a supersonic jet. It is much harder to get rid of the bad handling of the discharge, because its characteristics depend on the parameters of the gas in the area of activation, and to cope with the fact that a significant portion of the discharge of electrons is not involved in the activation process since energy of such elecnrons lie below the threshold for dissociation.

Thus, a technology conversion of light hydrocarbons in the gasoline fraction, based on plasma-chemical approach has not succeeded. The main reason, apparently, is the apparent "equilibrium" of processes in the flow of weakly ionized methane plasma. Activation of any kind of discharge leads to an increase of gas temperature in the flow, and heavy hydrocarbons which are produced with the participation of radicals formed in the discharge, will inevitably disintegrate soon after birth. This pessimistic conclusion was confirmed with both numerous experiments and calculations.

\section{Gas-Jet Plasma Chemictry}


The Institute of Thermophysics SB RAS has been patented [10] the idea to activate the supersonic gas jet reagents with highenergy electron beam. Using electron beam led to the fact that the distribution function of electron energies significantly shifted towards higher energies compared with the discharge, i.e. at the same injected power significantly increased the proportion of electrons with energies above the dissociation threshold of the flow particles. Experiments [11-13] have shown that the proposed method provides an efficient generation of radicals that have been used to produce hydrogen from methane, and for the deposition of silicon from silane coatings. Thus the high efficiency of the method was proved where it's enough to initiate rapid process of fragmentation reactions. However, attempts to use well-tried methods to the synthesis of heavy hydrocarbons did not give the expected result. Model calculations have also shown a low efficiency of this process: formed radicals are dying rapidly in binary reactions and increase the number of activating electrons leads not only to increase the number of radicals, but also stimulates the breakdown of already formed heavy molecules. Thus, to achieve this task it should be to found additional catalytic mechanisms that trigger the process of synthesis.

\section{Experiments in Conditions of Condensation}

It is well known that in a supersonic jet, due to a sharp drop of gas temperature downstream until the cryogenic temperature, the formation of clusters is possible. The forces that hold the molecules in the cluster can lead to changes tof activation and ionization hreshold energy of molecules in the cluster, and broaden the energy levels. A large retention time of particles in the cluster increases the probability of energy transfer. For example, in [14-15] have shown that when activated by an electron beam of supersonic jets of argon mixtures with methane, monosilane and other molecular additives highly efficient energy transfer effect occurs. This effect is detected at a certain stage and is due to the presence of condensation in the flow of mixed clusters. We seemed logical to evaluate the possibility of using clustered methane jets to increase the efficiency and controllability the plasma chemical jet synthesis of heavy hydrocarbons. Previously [16], we investigated the process of clustering in the absence of electronic activation. In this case was demonstrated the possibility of controlled formation of clusters, including mixed, with average sizes ranging from a few to thousands of atoms or molecules.

The activation of flow with clusters can cause the following processes:

a) flow heating, consequently, decrease condensate fraction, by reducing the concentration of clusters, and by decreasing their average size;

b) electron - stimulated condensation, in which the ionized particles becomes nuclei of clusters, so that the number of clusters and the fraction of condensate are increase;

c) electron stitching of molecules in the cluster, in which hydrogen atoms emitters due to the interaction with the electron beam with a cluster, and the remaining radicals are linked to the stable molecules of heavy hydrocarbons.

The research was performed using the LEMPUS experimental setup of Novosibirsk State University [16-17]. We used molecular beam mass spectrometry apparatus and activating electron gun. The working gas used natural gas composition: methane $\mathrm{CH}_{4}-94.5 \%$, ethane $\mathrm{C}_{2} \mathrm{H}_{6}-4.2 \%$, propane $\mathrm{C}_{3} \mathrm{H}_{8}-$ $1.1 \%$, butane $\mathrm{C}_{4} \mathrm{H}_{10}-0.2 \%$, pentane $\mathrm{C}_{5} \mathrm{H}_{12}-0.03 \%$. The stagnation pressure $\mathrm{P}_{0}$ varying from $1 \mathrm{kPa}$ to $10^{3} \mathrm{kPa}$. For activating of gas jet the focused electron beam with diameter 1-3 mm and electron energy 3-5 keV was used. Electron beam crossed the supersonic jet perpendicular to its axis at distance $5-20 \mathrm{~mm}$ from nozzle.

It is know that in the mixture flows condensation began from admixture of easy-to-condensing components [14]. Ethane and heavier hydrocarbons condense much better than methane. Therefore the number of ethane molecules in the cluster, at least at the initial stage of condensation, can significantly exceed the number of methane molecules. Activation of the jet by electrons leads primarily to an earlier beginning of condensation, as well as a greater drop in gas density due to expansion of the flow due to thermal heating

Figure 1 shows the overview pulsed mass spectra recorded at a fixed pressure of $1000 \mathrm{kPa}$ braking under normal conditions and with the activation. We see that the amplitude of the signal at mass peaks $\mathrm{CH}_{n}$ when you turn on the electron beam is significantly reduced. However, the masses of the dimers $\left(\mathrm{C}_{2} \mathrm{H}_{n}\right)$ drop is not as great as in the case of monomers, and the amplitude on the mass $\mathrm{m} / \mathrm{e}=41$ and $\mathrm{m} / \mathrm{e}=43$ even grow.

Condensation leads to release a stream of additional energy, resulting in the jet expands and the density at the axis decreases. In addition, the displacement of molecules from the jet axis by clusters also leads to a drop in the density of monomers, and the intensity of mass peaks of the monomers with increasing $\mathrm{P}_{0}$ even slightly reduced.

Thus, while the electron beam heats the gas, the formation of clusters in a supersonic flow occurs quite efficiently. Than larger the particle, than less effect on their quantity of activating electrons. We can assume that the electron energy is spent not on the collapse of large clusters, as, for example, for yhe formation of chemical bonds in the van der Waals particles.

The normalization of the intensities of mass peaks in the intensity of the peak $\mathrm{m} / \mathrm{e}=16$ (Figure 2) can reverse the effects associated with the fall of the density due to expansion of the flow, and more accurately determine the effect of activating the beam of electrons on the composition of the stream.

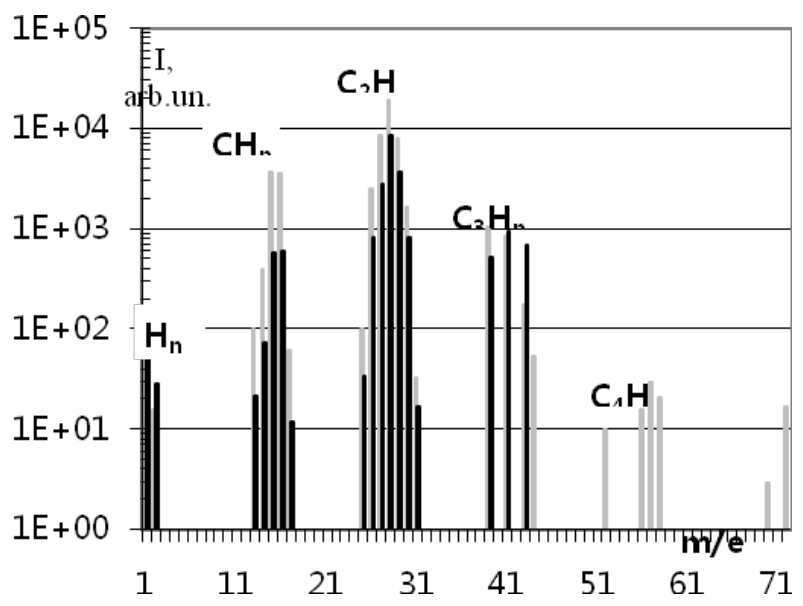

Figure 1. Mass spectra of natute gas with and without elrctron beam activation. 


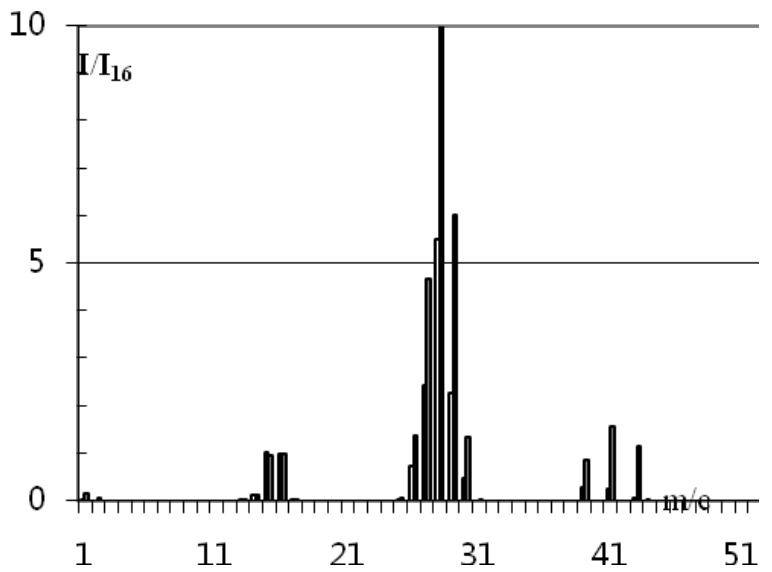

Figure 2. Dependence of the intensities of ion peaks from the stagnation pressure with electron activation (aeb) and without activation.

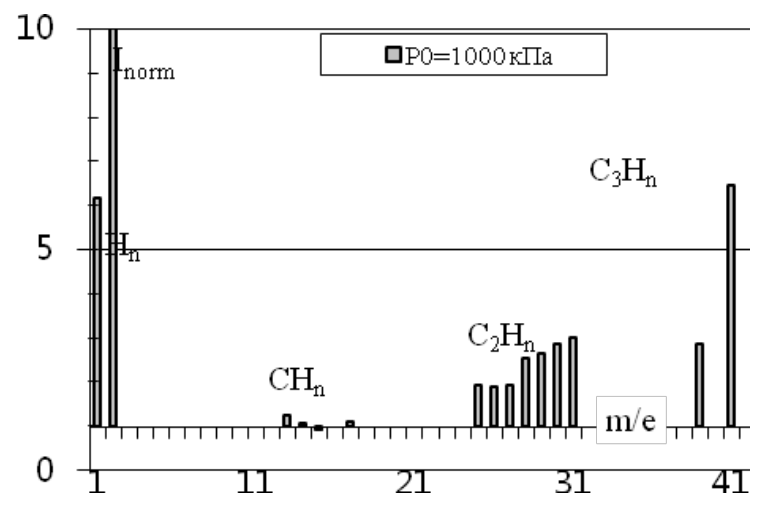

Figure 3. Dependence of the intensities of ion peaks from the stagnation pressure with electron activation (aeb) and without activation.

Found that the ratio of peak $\mathrm{m} / \mathrm{e}=15$ and $\mathrm{m} / \mathrm{e}=16$ when activated by electrons remains practically unchanged. Consequently, we can assume that the mass peak $\mathrm{m} / \mathrm{e}=15$, i.e. $\mathrm{CH}_{3}{ }^{+}$, is formed directly in the ionizer of the mass spectrometer for dissociative ionization of methane. The $\mathrm{CH}_{3}$ radical is formed by electron gun activation of methane jet, dying in the course of the reactions directly in the gas stream and to the sensor of the mass spectrometer does not reach. Ionized components of the plasma flow, including the ion $\mathrm{CH}_{3}{ }^{+}$, pass through a grounded conductive molecular beam skimmer system in small quantities, so the ability to contribute to the mass spectrometer signal from the ionized component of the flow is not considered.

Finally, the normalized relative intensities of mass peaks with ionization and without ionization $\mathrm{I}_{\text {norm }}=\left(\mathrm{I} / \mathrm{I}_{16}\right)_{\mathrm{aeb}} /\left(\mathrm{I} / \mathrm{I}_{16}\right)_{0}$, we obtain the "enrichment" - an amount reflecting the impact of activation on the intensity of the mass peak.

In Figure 3 shows that the effect of activating the flow by electrons does not affect the relative intensity of the peaks $\mathrm{CH}_{n}$, however, leads to a significant change in the other masses. The increase in the proportion of free hydrogen $\left(\mathrm{H}, \mathrm{H}_{2}\right)$, as well as the relative share of the complexes $\mathrm{C}_{2} \mathrm{H}_{n}, \mathrm{C}_{3} \mathrm{H}_{n}$, possibly a consequence of the restructuring of weakly bound van der Waals bonds in the hydrocarbon chemistry in large complexes.

Analysis of the experimental data shows the following. At the initial stage of condensation the activation of the jet gas by electron beam stimulates the clustering at lower stagnation pressures. At the same time, apparently, the heating of the gas flow proceed, which leads to a drop in gas density in the axial region. At higher stagnation pressures, i.e. on advanced stage of condensation, the number of heavy particles increases. One can suggest that the observed effect at high stagnation pressures due to the synthesis of heavy particles from the flow of methane is activated by an electron beam.

Thus, the efficiency of ion-cluster interactions in supersonic flows of hydrocarbons opens up the possibility of their use in GTL technology.

\section{Acknowledgements}

The work is performed with the financial support of the grant from the Russian government No. 11.G34.31.0046 for public support of scientific research under the guidance of leading scholars in Russian universities (leading scientist - K.Hanyalich, NSU) and by the Ministry of Education and Science of the Russia, project No. 1.22.05.

\section{REFERENCES}

[1] Huang Jian, Suib S. L. Dimerization of Methane through Microwave Plasmas // J. Phys. Chem., 1993. Vol. 97. P. 9403-9407.

[2] Eliasson B., Kogelschatz U., Killer E. Hydrogenation of Carbon Dioxide and Oxidation of Methane in an Electrical Discharge // Proc. 11th World Hydrogen Energy Conference, Stuttgart, Germany, 1996. P. 2449-2459.

[3] Fincke J.R., Anderson R.P., Hyde T.A.. Plasma Pyrolysis of Methane to Hydrogen and Carbon Black // Ind. Eng. Chem. Res., 2002. Vol. 41. P. 1425-1435.

[4] Kozlov K.V., Michel P., Wagner H.-E. Synthesis of organic compounds from $\mathrm{CH}_{4}-\mathrm{CO}_{2}$ - mixtures in barrier discharges with different dielectric materials // 14th Internat. Symp. on Plasma Chemistry, 1999. Vol. IV. P. 1849-1854.

[5] Kazuhisa Murata, Yoji Ushijima. A method of hydrogen producing // Patent JP No. 2767390 on 06/18/1998.

[6] Eliasson Baldur, Zhang Kui, Kogelschatz Ulrich. Synthesis of hydrocarbon fuel using electric discharge // Patent EP No. 1038855 on $27 / 09 / 2000$.

[7] Medvedev Yu.V., Remnev G.E., Smetanin V.I. The method of conversion of light hydrocarbons in the heavier // RF patent No. 2149884 on $01 / 06 / 1999$.

[8] Sirotkina E.E., Kudryashev S.V., Ryabov A.Yu. A method of producing isomeric structure hydrocarbons // RF patent No. 2123992 on 27/12/1998.

[9] Czernichowski Piotr, Czernichowski Albin. Conversion of hydrocarbons using the moving electric arcs in the presence of water vapor and / or carbon dioxide // Patent FR No. 2758317 on 17/07/1998.

[10] Sharafutdinov R.G., Karsten V.M., Polisan A.A. The method of homogeneous and heterogeneous reactions with the use of plasma // RF Patent No. 2200058 on 10/03/2003.

[11] Sharafutdinov R.G., Karsten V.M., Khmel S.Ya. et al. Epitaxial silicon films deposited at high rates by gas-jet electron beam plasma CVD // Surface and Coatings Technology, 2003. Vol. $174-175$. P. $1178-1181$.

[12] R. G. Sharafutdinov, A. E. Zarvin, V. Zh. Madirbaev, V. V. Gagachev, and G. G. Gartvich. Hydrogen production from me- 
thane in electron-beam-generated plasma // Technical Physics Letters. 2005. Vol. 31. No. 8. P. 641-643.

[13] V. A. Vinokurov, R. G. Sharafutdinov, Yu. I. Tychkov Plasma-chemical processing of natural gas // Chemistry and Technology of Fuels and Oils. 2005. Vol. 41. No. 2. P. 112-115.

[14] A. E. Zarvin, V. Zh. Madirbaev, N. G. Korobeishchikov, G. G. Gartvich, and R. G. Sharafutdinov. Effect of small methane and monosilane additives on clustering in pulse supersonic argon jets // Tech. Phys. 2005. Vol. 50. P. 1444-1450.

[15] V. Zh. Madirbaev, A. E. Zarvin. Ion-cluster excitation of atomic argon levels in molecular gas mixtures // Vestnik Novosibirsk State University. Series: Physics. 2007. Vol. 2. Issue 1. P. 36-43.

[16] N. G. Korobeishchikov, A. E. Zarvin, V. Zh. Madirbaev and R. G. Sharafutdinov. Condensation of argon, monosilane and their mixture in a pulse free jet // Plasma Chem. Plasma Proc. 2005. Vol. 25. P. 319-349.

[17] A. E. Zarvin, N. G. Korobeishchikov, V. V. Kalyada, V. Zh. Madirbaev. Formation of mixed clusters in a pulsed helium - oxygen - isoprene supersonic jet // Eur. Phys. J. D. 2008. Vol. 49. P. 101-110. 